\title{
Impact of Body Mass Index on the Initial In-Brace Correction in Patients With Idiopathic Scoliosis
}

\author{
Samra Pjanić, ${ }^{1}$ Goran Talić, ${ }^{1}$ Dragana Bojinović-Rodić ${ }^{1}$
}

\begin{abstract}
Background/Aim: Many factors affect initial in-brace correction and treatment outcome in patients with idiopathic scoliosis. Previous studies have observed contradictory results on the role of BMI in orthotic treatment. The aim of this study was to examine whether BMI impacts in-brace correction, isolated and in relation to other predictive factors (curve magnitude, curve location and Risser sign).

Methods: A retrospective study has been conducted on patients with idiopathic scoliosis treated with Cheneau-Sobernheim brace, that had no prior treatment. The collected and analysed data included patient demographics, BMI percentile and radiological parameters (curve magnitude in Cobb angle, curve location, Risser sign). The initial in-brace correction was expressed as a percentage of Cobb angle reduction in the brace as opposed to Cobb angle out of brace. Patients were categorised into groups according to their BMI, expressed in percentiles, ie: low BMI $(<5$ percentiles), normal BMI (5-85 percentiles) and high BMI ( $>85$ percentiles). To determine the significant difference and correlation relationship between the examined variables, variance tests, t-test with unequal variance, and Pearson correlation coefficient have been used.

Results: The cohort study comprised 213 patients (170 females and 43 males) with a mean age of 13.5 years at brace prescription. Low BMI has been detected in $10 \%$ patients, normal BMI in $78 \%$ and high BMI in $10 \%$ patients. No significant difference in in-brace correction has been found between BMI groups, nor has there been any significant correlation between BMI and in-brace correction. Regarding other factors, significant difference within BMI groups was found between in-brace correction and curve location, as well as in-brace correction and Risser sign. Lumbar curves had significantly better in-brace correction than thoracic curves. Significant correlations between in-brace correction and curve magnitude, curve location and Risser sign were detected.

Conclusion: The results of the present study show that, as an independent factor, BMI does not impact in-brace correction. Other factors, such as curve magnitude, curve location and Risser sign, play a more significant role in the orthotic treatment of patients with idiopathic scoliosis.
\end{abstract}

Keywords: Scoliosis; Body mass index; Brace; Correction.
(1) Institute for Physical Medicine and Rehabilitation "Dr Miroslav Zotovic", Banja Luka, the Republic of Srpska, Bosnia and Herzegovina.

Correspondence: SAMRA PJANIĆ

E: samra.pjanic@hotmail.com

\section{ARTICLE INFO}

Received: 9 November 2020 Revision received: 9 March 2021 Accepted: 9 March 2021

\section{Introduction}

Idiopathic structural scoliosis is a three-dimensional spine deformity with a lateral deviation of the spine in the frontal plane, a vertebral rotation in the transversal plane and an impaired sagittal profile. The aetiopathogenesis is as yet unclear and the cause is unknown. However, it is pre- 
sumed to be multifactorial ${ }^{1}$ and manifests solely in healthy children. It can occur at any age, especially during the growth spurt, where most progression is expected at the beginning of puberty. ${ }^{2}$ The diagnosis of scoliosis is based on anamnesis, clinical and radiological assessment. Conservative treatment of scoliosis includes observation, physical therapy and bracing. The aim of the treatment is to stop the curve progression - possibly even reducing it - and to avoid surgery. Recent studies have shown that it is also possible to obtain some amount of curve correction. ${ }^{3}$ Factors that have been suggested as possible determinants of a higher risk of scoliosis progression include positive family history, laxity of skin and joints (connective tissue defect), flattening of physiological thoracic kyphosis, angle of trunk rotation exceeding $10^{\circ}$, and growth spurt. ${ }^{4}$ Brace treatment is indicated for curves from 20 to $45 \pm 5$ degrees of Cobb angle in children that are skeletally immature and have growth potential. Initial in-brace correction represents a reliable parameter for brace quality ${ }^{5}$ and a prognostic factor for short-term results, ${ }^{6}$ as well as long-term results of orthotic treatment. ${ }^{7}$ In addition to well-defined prognostic factors - which affect initial in-brace correction ${ }^{8}$ and therefore treatment results - various studies explored the influence of Body Mass Index (BMI) on the initial in-brace correction and treatment outcome, with varying results.

The aim of this study was to examine whether BMI impacts initial in-brace correction in children with idiopathic scoliosis isolated and in relation to other prognostic factors (curve magnitude, curve location and Risser sign).

\section{Methods}

This was a retrospective study that included patients from the Institute for physical and rehabilitation medicine „Dr Miroslav Zotovic“ in Banja Luka, Republic of Srpska, Bosnia \& Herzegovina. The study has been approved by the Institute's Ethics Committee. All patients were diagnosed with idiopathic scoliosis and have been treated by the Team for scoliosis, which includes specialists for spinal deformities, orthotists and therapists. The treatment of these patients is based on the SOSORT guidelines for conservative treatment of scoliosis $(2016)^{1}$ and standardised treatment protocols. In this facility, the type of brace being prescribed and manufactured is Cheneau-Sobernheim. This study only included patients who had no prior brace treatment. In patients with one or more compensatory curves, the focus was on the primary scoliotic curve.

The analysed data was obtained from the internal database, the hospital information system KIS, including patient demographics, BMI in percentiles and radiological parameters (on the X-ray image at brace prescription and on in-brace X-ray, one month after the final brace application). Radiological parameters include magnitude of the primary curve (measured by Cobb's angle at out- and inbrace X-ray), location of the primary curve, and Risser stage. The location of the primary curve is classified into the following categories: thoracic, thoracolumbar and lumbar.

The initial in-brace correction is calculated as a reduction of Cobb angle of the primary curve in brace and out of brace, expressed as a percentage of the Cobb angle reduction in brace.

The BMI is evaluated differently in children then in adults, given that children's bodies change rapidly. This is why the standardised formula for children, ages 5 through 18 years, is based on height, weight and the patient's age. In this study, the authors looked at these parameters at brace application.

Patients have been categorised into groups according to their BMI, expressed in percentiles: low BMI ( $<5$ percentiles), normal BMI (5-85 percentiles) and high BMI ( $>85$ percentiles).

To examine the effect of the BMI on in-brace correction, the correlation between BMI and initial in-brace correction in total and according to BMI groups has been tested. Other relevant factors, which affect in-brace correction, have also been taken into consideration, such as: curve magnitude of the primary curve measured by Cobb angle, location of the primary curve and Risser sign.

For the statistical analysis, the program SPSS Statistics for Windows 21.0 has been used. To test whether significant differences exist between the examined variables and BMI groups, variance tests (ANOVA) and t-test with unequal variance have been performed. To test the correlations between BMI and other variables, Pearson correlation coefficient was used. The level of significance has been set to $\mathrm{p}<0.05$. 


\section{Results}

Out of 213 patients, 170 were females (79.8\%) and 43 males (20.2\%). The mean age at brace prescription was 13.5 (SD \pm 2.4 , range 5 to 17.5 ) years. The mean BMI was 44.2 percentiles (SD \pm 29.74). The results, according to groups, were as follows: low BMI has been detected in 21 patients (10\%), mid-BMI in 167 patients (78\%) and high BMI in 25 patients (12\%). The mean magnitude of the primary curve was $29.53^{\circ}$ of Cobb angle (SD \pm 9.2 , range $13-59^{\circ}$ ). The mean Risser sign was 1.65 (SD \pm 1.4 , range 0 to 4 ). The mean initial inbrace correction was $53.5 \%$ (SD $\pm 24.6,0-100 \%$ ).

Table 1 shows the number of patients according to BMI groups, as well as mean values of their age at brace prescription, radiological parameters (curve magnitude and Risser sign) and in-brace correction.

Table 1: Patients' mean values $( \pm S D)$ of age, radiological parameters and in-brace correction according to BMI groups

\begin{tabular}{lcccccc}
$\begin{array}{l}\text { BMI } \\
\text { groups* }\end{array}$ & $\begin{array}{l}\text { Number of } \\
\text { patients }\end{array}$ & $\%$ & $\begin{array}{l}\text { Age at brace } \\
\text { prescription } \\
\text { (in years) }\end{array}$ & $\begin{array}{l}\text { Risser } \\
\text { sign }\end{array}$ & $\begin{array}{l}\text { Curve } \\
\text { magnitude }\left({ }^{\circ}\right)\end{array}$ & $\begin{array}{l}\text { In-brace } \\
\text { correction (\%) }\end{array}$ \\
\hline Low BMI & 21 & 10 & $14.5( \pm 2.43)$ & $1.7( \pm 1.56)$ & $32.4 \quad( \pm 9.72)$ & $46.7( \pm 21.2)$ \\
\hline Mid BMI & 167 & 78 & $13.8( \pm 2.24)$ & $1.8( \pm 1.44)$ & $29.2 \quad( \pm 8.96)$ & $54.8( \pm 26.2)$ \\
\hline High BMI & 25 & 12 & $11.5( \pm 2.78)$ & $0.8( \pm 1.22)$ & $29.5( \pm 10.45)$ & $50.8( \pm 26.8)$ \\
\hline
\end{tabular}

*Low BMI < 5 percentiles; Mid BMI 5-85 percentiles; High BMI > 85 percentiles

\section{$\mathrm{BMI}$ and in-brace correction}

Figure 1 illustrates the mean values (\%) of the initial in-brace correction in total and according to BMI groups. Even though the greatest in-brace correction has been detected in the mid-BMI group (54.79\%), and the least in the low-BMI group (46.73\%), the difference is not significant $(\mathrm{F}=1.18, \mathrm{p}=0.31)$.

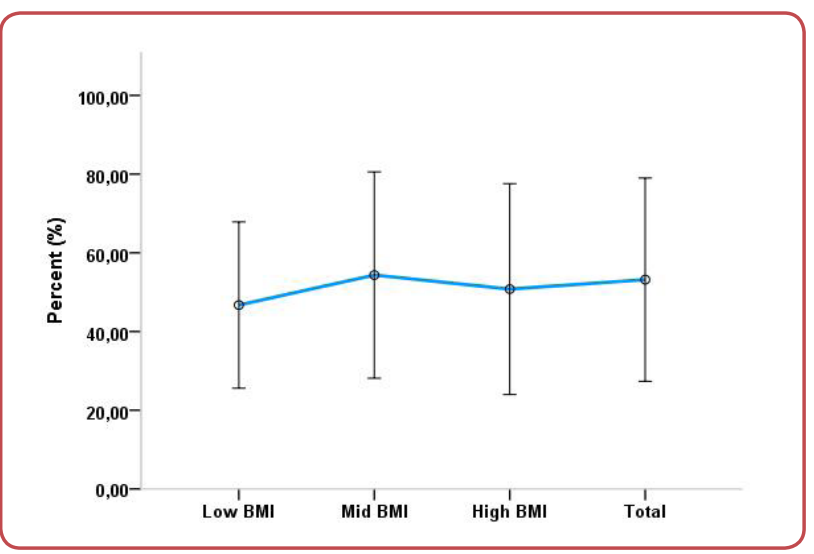

Figure 1: Mean values ( $\pm S D$ ) of in-brace correction (\%) in total and according to BMI groups

Figure 2 shows the results of the correlation between BMI, expressed in percentiles, and in-brace correction. It depicts a negative correlation that is not statistically significant $(r=-0.061, p=0.38)$.

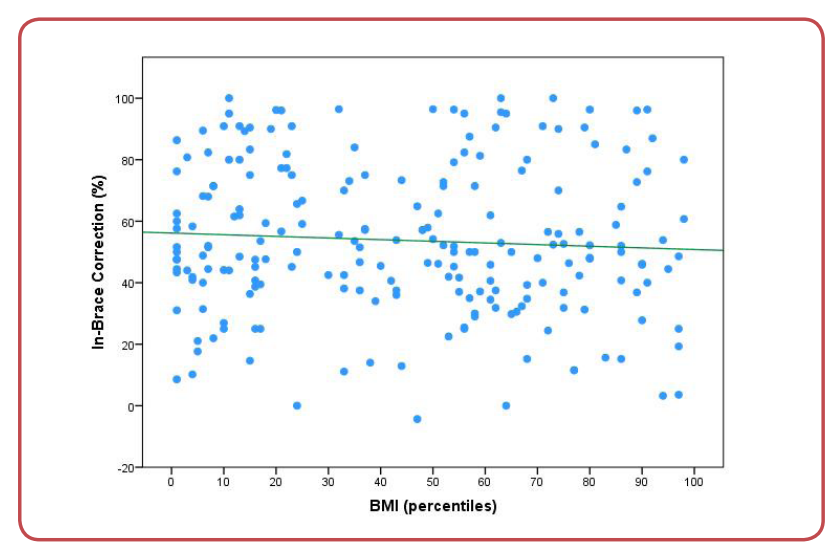

Figure 2: Correlation between BMI and in-brace correction

\section{Curve magnitude and in-brace correction}

Figure 3 displays the mean values of curve magnitude $\left({ }^{\circ}\right)$ and in-brace correction in total and according to BMI groups.

There was no significant difference in curve magnitude between BMI groups ( $F=1.13 ; p=0.33)$. There is a significant negative correlation between curve magnitude and in-brace correction $(r=-0.399, p=0.00)$. There is a better correlation in high- $(\mathrm{r}=-0.622, \mathrm{p}=0.001)$ and low-BMI groups $(r=-0.615, p=0.003)$, compared to the mid-BMI group $(r=-0,402, p=0,00)$. 


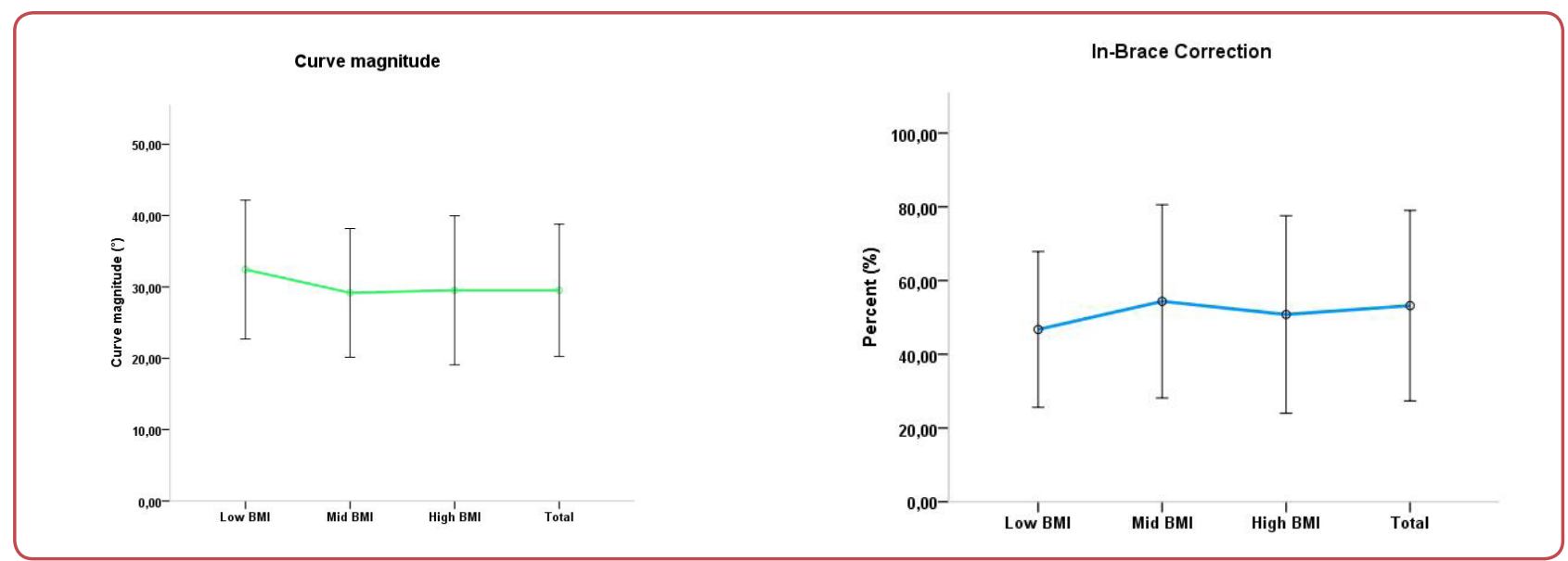

Figure 3: Mean values $( \pm S D)$ of curve magnitude and in-brace correction in total and according to BMI groups

\section{Curve location and in-brace correction}

Figure 4 shows the in-brace correction in BMI groups according to the location of the primary curve.

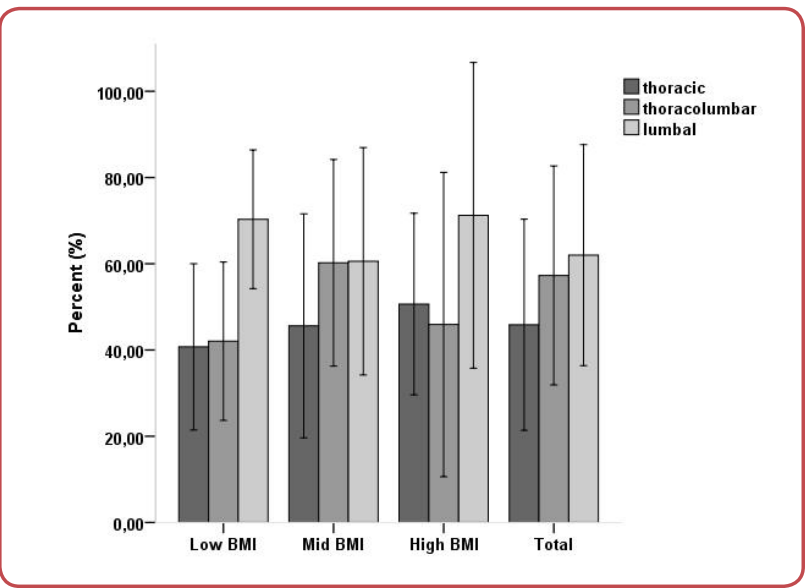

Figure 4: Mean values $( \pm S D$ ) of in-brace correction for different curve locations in total and according to BMI groups
The location of the primary curve is thoracic in 94 patients (44.1\%), thoracolumbar in 76 patients (35.7\%) and lumbar in 43 patients $(20.2$ $\%)$. There was a significant difference in the inbrace correction between groups that are categorised according to the location of the primary curve $(F=7.59, p=0.001)$.

A better in-brace correction was observed in patients with primary lumbar curves (62\%), as opposed to patients with primary thoracic curves $(46 \%)$ in the low- $(p=0.035)$ and mid-BMI group $(\mathrm{p}=0.012)$. There were no significant differences in the correction between thoracolumbar and lumbar curves $(\mathrm{p}=0.588)$.

A significant correlation can be observed between in-brace correction and location within the low$(\mathrm{F}=4.006, \mathrm{p}=0.036)$ and mid-BMI group $(\mathrm{F}=$ $6.653, p=0.002)$. The correlation in the high-BMI group was not significant $(F=0.698, p=0.508)$.

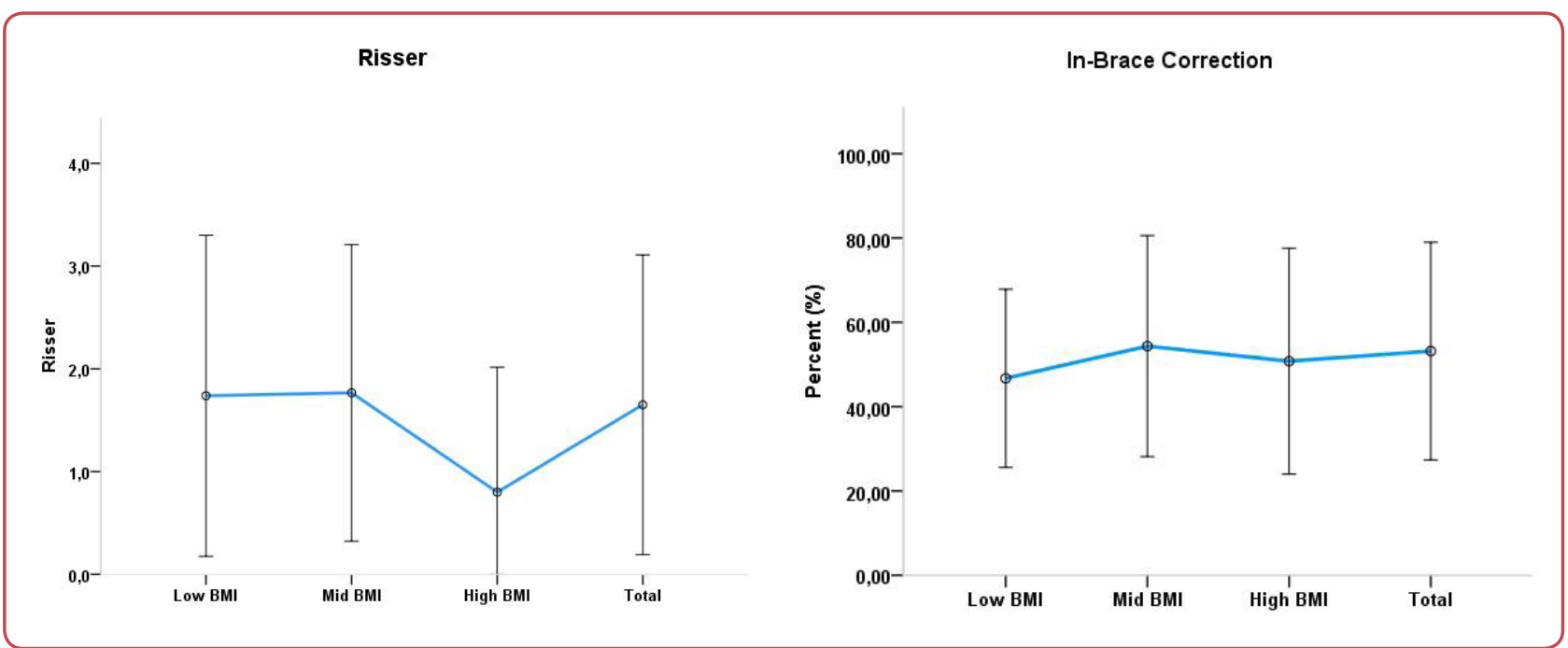

Figure 5: Mean values $( \pm S D)$ of in-brace correction for different curve locations in total and according to BMI groups 


\section{Risser sign and in-brace correction}

Figure 5 depicts mean values of the Risser sign and in-brace correction (\%) in total and according to BMI groups.

A significant difference in the Risser sign between BMI groups $(F=5,001, p=0.008)$ has been identified, especially between mid- and high-BMI groups $(F=5.01 ; p=0.01)$.

A lower Risser sign in the high-BMI group can be explained by the patients' age, who were significantly younger compared to the other two groups $(\mathrm{F}=11.45 ; \mathrm{p}=0.00)$.

There was a significant negative correlation between in-brace correction and Risser sign ( $\mathrm{r}=$ $-0.203, p=0.003)$. Within group, this correlation was only significant for mid-BMI group $(r=-0.250$, $\mathrm{p}=0.001)$.

\section{Discussion}

Bracing proved to be effective in the treatment of idiopathic scoliosis. ${ }^{9}$ In-brace correction is considered to be one of the most important parameters for brace quality assessment, but it can also be a predictor for short-term and long-term treatment outcomes in patients with idiopathic scoliosis. In the literature, currently reported percentages of in-brace correction vary from $20-25 \%$ to $40-50$ $\%$. This depends on various factors, such as curve magnitude and location, Risser sign, curves exceeding $30^{\circ}$, vertebral rotation, compliance and BMI. ${ }^{10}$ It is suggested that the in-brace correction must be at least 30-50\% in order to prevent significant curve progression. ${ }^{11}$ In his study, Landauer reported that a good initial correction, of over $40 \%$ in a Cheneau type brace, had significant impact on the final outcome. ${ }^{12}$ According to another study ${ }^{13}$ insufficient in-brace correction is associated with poor treatment outcome. For a successful orthotic treatment of patients with idiopathic scoliosis, it is significant to understand how different factors affect in-brace correction. ${ }^{14}$

The primary objective of the present study has been to determine whether the BMI significantly impacts in-brace correction in patients with idiopathic scoliosis, as well as whether it can be considered one of the predictive factors for treatment outcome. Previous studies on the influence of BMI on in-brace correction and outcome have showed contradictory results. High BMI is considered a negative predictive factor for brace effectiveness. It has been reported that patients with a high BMI have a 3.1-fold greater likelihood of unsuccessful bracing than normal-weight patients. ${ }^{15}$ This is explained by the reduced action of corrective forces on the spine over the ribs due to excessive adipose tissue. These findings have been confirmed by Goodbody and colleagues ${ }^{16}$ where such poor treatment outcome in high-BMI patients is associated with insufficient in-brace correction and compliance. Furthermore, it has been found that a low BMI is an independent risk factor for poor outcome. It should be noted that in this study different range was set in low-BMI category $(<20$ percentiles). Similar results were observed in the study of Weixiang and colleagues ${ }^{17}$ who concluded that low BMI could predict brace failure. Lori and colleagues ${ }^{18}$ likewise concluded that a low BMI has the highest progression and the greatest surgical risk, although they were more complaint than high-BMI patients.

The results of present study showed that the BMI has no significant impact on in-brace correction in patients with idiopathic scoliosis. Therefore, it cannot be considered a predictive factor for the final outcome of bracing treatment, given the significance of immediate in-brace correction in predicting long-term results. ${ }^{19}$

The present findings are in accordance with those of Zaina and colleagues. ${ }^{20}$ They reported that high BMI cannot be considered a negative predictive factor for treatment outcome, due to insignificant differences in in-brace correction between midand high-BMI group. Their study concluded that type of treatment, brace type and efficiency play a key role in stopping the curve progression.

Even though a negative correlation between BMI and in-brace correction can be observed, it is not significant. The mean in-brace correction in these patients can be considered sufficient for successful outcome by the current standards (53\%). The lowest in-brace correction has been identified in the low-BMI group, which can be explained by older age, higher curve magnitude and Risser sign, with significant difference compared to other groups. This group also included more thoracic curves that show lower in-brace correction than 
lumbar curves. Nevertheless, there is no significant difference in in-brace correction between the low-BMI group compared to the other two groups.

In this study, other factors, such as curve magnitude and location, play a more critical role in the in-brace correction. There is a significant negative correlation between curve magnitude and inbrace correction, especially in the low- and highBMI groups. There is also a significant correlation between the location of the primary curve and inbrace correction in the low- and mid-BMI group. In both groups, better correction was achieved in lumbar curves, compared to thoracic curves, while no difference has been identified between thoracolumbar and lumbar curves.

Even though a significant negative correlation can be observed between in-brace correction and Risser sign, it only occurs in the mid-BMI group. Limitations of this study include its retrospective nature and inability to take into consideration other important factors associated with in-brace correction and treatment outcome - such as adherence to treatment and compliance - due to lack of appropriate objective monitoring. Dose response to bracing has been earlier confirmed ${ }^{21}$ concluding that wearing a brace a greater number of hours correlated with lack of curve progression and vice versa. Hours of bracing is associated with successful orthotic treatment. ${ }^{22}$

In conclusion, the results of this study show that the BMI, as an independent factor, does not impact initial in-brace correction. Other factors, such as curve magnitude, curve location and Risser sign, play a more significant role in the orthotic treatment of patients with idiopathic scoliosis. There are also other important factors that affect inbrace correction, such as compliance, that authors were not able to monitor. Even though some studies report that the BMI plays a significant role in treatment outcome, from these results it can be concluded that BMI cannot be considered as relevant prognostic factor for in-brace correction and treatment outcome.

\section{Acknowledgements}

None.

\section{Conflict of interest}

None.

\section{References}

1. Negrini S, Donzelli S, Aulisa AG, Czaprowski D, Schreiber $S$ et al. 2016 SOSORT guidelines: orthopaedic and rehabilitation treatment of idiopathic scoliosis during growth. Scoliosis 13, 3 (2018). https://doi. org/10.1186/s13013-017-0145-8.

2. Grivas TB, Vasiliadis E, Mouzakis V, Mihas C, Koufopoulos G. Association between adolescent idiopathic scoliosis prevalence and age at menarche in different geographic latitudes. Scoliosis 2006 May 23;1:9. doi: 10.1186/1748-7161-1-9.

3. Negrini S, Atanasio S, Fusco C, Zaina F. Effectiveness of complete conservative treatment for adolescent idiopathic scoliosis (bracing and exercises) based on SOSORT management criteria: results according to the SRS criteria for bracing studies - SOSORT award 2009 winner. Scoliosis 2009 Sep 4;4:19. doi: 10.1186/17487161-4-19.

4. Upadhyay SS, Nelson IW, Ho EK, Hsu LC, Leong JC. New prognostic factors to predict the final outcome of brace treatment in adolescent idiopathic scoliosis. Spine 1995;20(5):537-45.

5. Noonan KJ, Weinstein SL, Jacobson WC, Dolan LA. Use of the Milwaukee brace for progressive idiopathic scoliosis. J Bone Joint Surg Am 1996;78(4):557-67.

6. Zaina F, Donzelli S, Luisini M, Negrini S. Correlation between in-brace radiographic correction and short time brace results. Stud Health Technol Inform 2012;176:342-5.

7. Clin J, Aubin C-É, Sangole A, Labelle H, Parent S. Correlation between immediate in-brace correction and biomechanical effectiveness of brace treatment in adolescent idiopathic scoliosis. Spine (Phila Pa 1976) 2010 Aug;35(18):1706-13.

8. Chuandong L , Zifang H , Wenyuan S, Muping D , Shao$\mathrm{fu} \mathrm{H}$ et al. Factors that influence in-brace correction in patients with adolescent idiopathic scoliosis. World Neurosurg 2019;123:597-603.

9. Weinstein LS, Dolan L, Wright JG, Dobbs MB. Effects of bracing in adolescents with idiopathic scoliosis. N Engl J Med 2013;369:1512-21.

10. El Hawary R, Zaaroor-Regev D, Floman Y, Loonner BS, Alkhalife YI, Betz RR. Brace treatment in adolescent idiopathic scoliosis: risk factors for failure - a literature review. Spine J 2019;19(12):1917-25.

11. Emans JB, Kaelin A, Bancel P, Hall JE, Miller ME. The Boston bracing system for idiopathic scoliosis. Follow-up results in 295 patients. Spine (Phila Pa 1976) 1986;11(8):792-801.

12. Landauer F, Wimmer C, Behensky H. Estimating the final outcome of brace treatment for idiopathic thoracic scoliosis at 6-month follow-up. Pediatr Rehabil 2003;6 (3-4):201-7.

13. Van den Bogaart M, Van Royen JB, Haanstra TM, De Kleuver M, Sayf F. Predictive factors for brace treatment 
outcome in adolescent idiopathic scoliosis: a best-evidence synthesis. Eur Spine J 2019 Mar;28(3):511-25.

14. Chuandong L, Zifang H , Wenyuan S , Muping D, Shao$\mathrm{fu} \mathrm{H}$, et al. Factors that influence in-brace correction in patients with adolescent idiopathic scoliosis. World Neurosurg 2019 Mar;123:597-603.

15. O'Neill P, Lori K, Shindle M, Elerson E, Brintzenhofeszoc $\mathrm{K}$, Katz D et al. Decreased orthotic effectiveness in overweight patients with adolescent idiopathic scoliosis. J Bone Joint Surg 2005; 87(5):1069-74.

16. Goodbody CM, Asztalos IB, Sankar WN, Flynn JM. It's not just the big kids: both high and low BMI impact bracing success for adolescent idiopathic scoliosis. J Child Orthop 2016;10(5):395-404.

17. Weixiang S, Minghui S, Xiaodong Q, Zhou J. Low body mass index can be predictive of bracing failure in patients with adolescent idiopathic scoliosis: a retrospective study. Eur Spine J 2017 Jun;26(6):1665-69.

18. Lori AK, Wingfield JJ, Virostek D, Felton K, ChanHee J.
The Influence of Body Habitus on Documented Brace Wear and Progression in Adolescents With Idiopathic Scoliosis. J Pediatr Orthop 2020 Mar;40(3):171-5.

19. Clin J, Aubin C-É, Parent S. Biomechanical simulation and analysis of scoliosis correction using a fusionless intravertebral epiphyseal device. Spine 2015;40(6):369-76.

20. Zaina F, Donzelli S, Negrini S. Overweight is not predictive of bracing failure in adolescent idiopathic scoliosis: results from a retrospective cohort study. Eur Spine J 2017 Jun;26(6):1670-1675.

21. Katz DE, Herring JA, Browne RH, Kelly DM, Birch JG. Brace wear control of curve progression in adolescent idiopathic scoliosis. J Bone Joint Surg Am 2010 June;92(6):1343-52.

22. Rahman T, Borkhuu B, Littleton AG, Sample W, Moran $\mathrm{E}$ et al. Electronic monitoring of scoliosis brace wear compliance. J Child Orthop 2010;4(4):343-7. 